\section{Las muchas dimensiones de la equidad en la salud}

\author{
Juan Antonio Casas-Zamora ${ }^{1}$ \\ y Davidson R. Gwatkin ${ }^{2}$
}

1 División de Salud y Desarrollo Humano, Organización Panamericana de la Salud, Washington, D.C., Estados Unidos de América.

2 Banco Mundial, Washington, D.C., Estados Unidos de América.
Los artículos que se presentan en este número de la Revista Panamericana de Salud Pública/Pan American Journal of Public Health ilustran claramente las muchas dimensiones que tiene la equidad en el campo de la salud. Su contenido constituye una revisión muy especial de los temas que actualmente se debaten en relación con los aspectos conceptuales y metodológicos del campo y las pruebas empíricas que existen en torno a los factores que determinan la inequidad en materia de salud. Los escritos que en él se presentan comienzan con el sólido argumento planteado por Amartya Sen, quien pide que la inequidad en el campo de la salud se considere no solo un fenómeno aislado, sino más bien una manifestación de inequidades sociales de mayor alcance. A continuación se resumen los resultados de diversos proyectos de investigación patrocinados por la Organización Panamericana de la Salud (OPS) sobre las dimensiones de las inequidades en salud y sus factores determinantes en el continente americano. En una serie de ensayos se examinan varios temas relacionados con la equidad, entre ellos el género, la etnia, la edad, la pobreza, la distribución de ingresos y el crecimiento económico, la gobernancia, la globalización, la legislación, la ética y el papel del acceso a información y al financiamiento. En los ensayos también se contemplan nuevas maneras de evaluar las consecuencias sanitarias de las políticas públicas encaminadas a fomentar la equidad en materia de salud y formas novedosas de transferir información y conocimientos de los investigadores a las autoridades decisoras. Otra contribución importante es la presentación de un creciente número de fuentes de información internéticas que han sido creadas por la OPS y otros organismos. Estos materiales nuevos están llevando al desarrollo de una biblioteca virtual mundial sobre la equidad en materia de salud que servirá para estrechar los lazos entre el mundo académico, los gobiernos y la sociedad civil, que sin excepción han manifestado su interés y compromiso para crear un mundo donde haya más equidad y justicia en el ámbito de la salud.

De los temas abordados en los escritos que integran este número, ¿cuáles son los más importantes? Cada lector tendrá, indudablemente, su propia respuesta a esta pregunta, pero en toda probabilidad, la respuesta abarcará cuando menos uno o varios de los siguientes aspectos:

- Las disparidades de carácter económico, por importantes que sean, representan solamente una de las numerosas causas importantes de que haya diferencias poco equitativas en la salud de diferentes grupos. La importancia de las disparidades de carácter económico queda destacada en el artículo de Dachs et al., que versa sobre la salud y la búsqueda de atención sanitaria según datos extraídos de encuestas de hogares. Pero si alguien piensa que las diferencias de tipo económico son las únicas que cuentan o las que ejercen un peso mayor, es de rigor que lean otros trabajos: el artículo de Gómez sobre las disparidades de género, el planteamiento de Larrea y Freire sobre las diferencias de orden étnico en relación con la desnutrición, y la presentación de Soares et al. sobre las diferencias observadas en zonas urbanas y rurales en el acceso a agua potable. Resulta evidente que las diferencias relacionadas con el género, el origen étnico y la situación geográfica revisten tanta importancia como las de carácter económico.

- La medición de las desigualdades usando medidas estándar, tales como las tasas de mortalidad y el uso de servicios de salud, captan solamente una pequeña fracción del panorama total de la inequidad en materia de salud. Las desigualdades en el campo de la salud no pueden desligarse de otras inequidades de tipo social. Este mensaje, que Sen resalta justificadamente, también se trasluce en otros artículos. Como apuntan Soares et al., el abastecimiento de agua potable se encuentra en el margen de la definición tradicional del sector de la salud, pero no por ello es menos importante. Asimismo, el tema central del trabajo de Larrea y Freire sobre la desnutrición infantil posee la virtud de recordarnos la importancia de mirar más allá de los indicadores estándar de las condiciones de salud y de tener en cuenta otros parámetros, tales como el estado nutricional. Otra medida importante consiste en introducir el enfoque de equidad en la vigilancia y análisis epidemiológicos y en la vigilancia y análisis de la situación de salud autopercibida, utilizando fuentes de información de base poblacional, como las encuestas de hogares descritas por Dachs. Se trata de una medida que mejorará notablemente la capacidad del sector para realizar intervenciones más eficaces y mejor enfocadas, así como para abogar por intervenciones que lleven a una mayor equidad. 
- No todas las disparidades en el ámbito de la salud encierran una falta de equidad. La clásica definición de la inequidad en el campo de la salud es una disparidad o desigualdad innecesaria, evitable e injusta. Si bien es cierto que muchas desigualdades cumplen estos tres criterios, no todas lo hacen. Esto se hace especialmente patente en las discusiones sobre el género que hacen Gómez y Sen en sus artículos. La ventaja que poseen las mujeres en virtud de que viven más tiempo no refleja necesariamente una falta de equidad, ya que es posible que esta diferencia se deba a factores biológicos y no a causas que puedan prevenirse mediante intervenciones dictadas por políticas. Como señala Gómez, las mujeres latinoamericanas muestran un mayor uso de servicios de salud, en términos porcentuales, que los hombres. No obstante, esto representa una desigualdad que es justa, ya que tiende a compensar la mayor morbilidad que sufren las mujeres. Por otra parte, en casi todos los países de la Región, las mujeres destinan una mayor proporción de sus gastos de bolsillo a servicios y productos para la salud que los hombres. Claramente, esta situación encierra una falta de equidad y debe subsanarse con políticas específicas. Ejemplos como estos demuestran la necesidad de reflexionar detenidamente antes de conlcuir que una desigualdad constituye una falta de equidad.

- Diferentes grupos poblacionales perciben su salud de un modo distinto. Esto se aprecia en los trabajos de Gómez y Dachs. Según Gómez, las mujeres en los países encuestados suelen notificar problemas de salud con mayor frecuencia que los hombres. Este tema ocupa un lugar aun más prominente en el trabajo de Dachs, quien examina por estratos socioeconómicos el estado de salud indicado por el propio paciente (self-reported health status, SRHS) y encuentra que los ricos a menudo se sienten tan enfermos como los pobres. Este hallazgo, que coincide con los de otros estudios en que el estado de salud ha sido autoevaluado, es lo contrario del que apunta a una morbilidad y mortalidad mucho mayores entre los pobres, como han demostrado los exámenes de los registros de defunción y la determinación objetiva del estado de salud mediante examen físico y de laboratorio. Esto indica que los ricos y pobres tienen expectativas diferentes. Es decir, lo que una persona con más recursos económicos percibe como una serie de síntomas debilitantes, para un pobre es un estado normal sin importancia y parte ordinaria de su experiencia cotidiana. Es tentador interpretar esta disparidad como muestra del peligro de depender del SRHS, pero este último es importante, tan solo por sus implicaciones en lo que respecta a la adjudicación de recursos. Los ricos se perciben a sí mismos como enfermos y exigen y reciben una elevada proporción de los servicios de salud que, si se adjudicaran según criterios epidemiológicos establecidos externamente, deberían destinarse principalmente a los desfavorecidos. Si los ricos no fuesen desmesuradamente sensibles a su estado de salud y los pobres supieran cuán enfermos están en realidad, sería más fácil lograr la equidad en materia de salud.

- Los factores que determinan la inequidad en salud deben ser subsanados mediante políticas generales transcetoriales y mediante intervenciones apropiadas encaminadas a reducir la discriminación y las disparidades injustas. La mayor causa de inequidad en el campo de la salud sigue siendo, con mucho, las marcadas diferencias que aún se observan en la distribución de bienes y servicios esenciales. Estos patrones desiguales de acceso a los medios para satisfacer necesidades básicas y ejercer control sobre la vida personal se ven definidos por las relaciones intrafamiliares entre los sexos y personas de distinta edad, y en la sociedad más extendida por la distribución del ingreso, el estado sicioeconómico, la procedencia étnica, la escolaridad y la ubicación geográfica. El sector de la salud puede hacer mucho por remediar las consecuencias sanitarias más patentes de las disparidades sociales y económicas más amplias. A la larga, sin embargo, las políticas que mejoren a todos los desfavorecidos tendrán un impacto mucho mayor. Los nexos entre el crecimiento económico, la pobreza y la distribución del ingreso en América Latina y el Caribe son analizadas por Casas. El enfoque descrito por Barnes y Scott-Samuel para evaluar el impacto sobre la salud representa una de las maneras en que el sector sanitario puede lograr que otras entidades afines dedicadas al desarrollo en los niveles local y nacional participen activamente en la formulación y adopción de políticas públicas destinadas a fomentar la equidad. Otro enfoque para lograr este objetivo consiste en adoptar instrumentos políticos que permitan mayor equidad en el financiamiento de los servicios y en el acceso a ellos, tal como se describe en los trabajos de Suárez y Gwatkin.

Los temas que figuran en la lista anterior no simplifican los retos que enfrentan las personas que desean lograr una mayor equidad. No obstante, es necesario tener conciencia de estos temas para no caer en la adopción de enfoques demasiado simplistas y hasta contraproducentes. Los autores de los trabajos publicados en este número de la Revista Panamericana de Salud Pública/Pan American Journal of Public Health merecen nuestragratitud por compartir con nosotros sus sapiencias y ayudarnos a tomar las mejores decisiones posible en este campo tan difícil. 